\title{
Sialylation of IgG Fc domain impairs complement-dependent cytotoxicity
}

\author{
Isaak Quast, ${ }^{1}$ Christian W. Keller, ${ }^{1}$ Michael A. Maurer, ${ }^{1}$ John P. Giddens, ${ }^{2}$ Björn Tackenberg, ${ }^{3}$ Lai-Xi Wang, ${ }^{2}$ Christian Münz, ${ }^{4}$ \\ Falk Nimmerjahn, ${ }^{5}$ Marinos C. Dalakas, ${ }^{6}$ and Jan D. Lünemann ${ }^{1,7}$ \\ IInstitute of Experimental Immunology, Laboratory of Neuroinflammation, University of Zürich, Zürich, Switzerland. 'Department of Chemistry and Biochemistry, University of Maryland, College Park, \\ Maryland, USA. ${ }^{3}$ Department of Neurology, Philipps University of Marburg, Marburg, Germany. ${ }^{4}$ Institute of Experimental Immunology, Laboratory of Viral Immunobiology, University of Zürich, Zürich, \\ Switzerland. ${ }^{5}$ Department of Biology, Institute of Cenetics, University of Erlangen-Nürnberg, Erlangen, Cermany. ${ }^{6}$ Department of Neurology, Thomas Jefferson University, Philadelphia, Pennsylvania, USA \\ ${ }^{7}$ Department of Neurology, University of Basel, Basel, Switzerland.
}

\begin{abstract}
IgG molecules exert both pro- and antiinflammatory effector functions based on the composition of the fragment crystallizable (Fc) domain glycan. Sialylated IgG Fc domains have antiinflammatory properties that are attributed to their ability to increase the activation threshold of innate effector cells to immune complexes by stimulating the upregulation of the inhibitory Fcy receptor IIB (FcyRIIB). Here, we report that IgC Fc sialylation of human monoclonal IgC1 molecules impairs their efficacy to induce complement-mediated cytotoxicity (CDC). Fc sialylation of a CD20-targeting antibody had no impact on antibodydependent cellular cytotoxicity and did not change the affinity of the antibody for activating Fcy receptors. In contrast, the presence of sialic acid abrogated the increased binding of C1q to Fc-galactosylated IgC1 and resulted in decreased levels of C3b deposition on the cell surface. Similar to monoclonal antibodies, sialic acid inhibited the increased C1q binding to galactosylated Fc fragments in human polyclonal IgG. In sera derived from patients with chronic inflammatory demyelinating polyneuropathy, an autoimmune disease of the peripheral nervous system in which humoral immune responses mediate tissue damage, induction of IgC Fc sialylation was associated with clinical disease remission. Thus, impairment of CDC represents an FcyRindependent mechanism by which Fc-sialylated glycovariants might limit proinflammatory lgG effector functions.
\end{abstract}

\section{Introduction}

IgG molecules can trigger both pro- and antiinflammatory responses mediated by their fragment crystallizable domain (Fc). Proinflammatory pathways include the activation of innate immune effector cells via cellular receptors specific for the antibody constant region ( $\mathrm{F} c \gamma$ receptors, herein referred to as $\mathrm{Fc} \gamma \mathrm{Rs}$ ) and the activation of the complement system. Activation of the classical complement pathway via $\mathrm{C} 1 \mathrm{q}$ binding to human IgG1 and IgG3 molecules generates proinflammatory anaphylotoxins $\mathrm{C} 3 \mathrm{a}$ and $\mathrm{C} 5 \mathrm{a}$, which can trigger innate immune effector cell recruitment, and deposition of $\mathrm{C} 3 \mathrm{~b}$ on target cells enables their recognition through $\mathrm{C} 3 \mathrm{~b}$ receptors expressed on phagocytic, antigen-presenting cells $(1,2)$. Compared with the aforementioned effector functions that establish and maintain tissue inflammation, our understanding how IgG contributes to the resolution of inflammation is still vague.

Recent studies provided evidence that carbohydrates in the sugar moiety attached to the IgG Fc domain are essential for IgG functionality and its antiinflammatory capacity (3). IgG Fc contains a single, highly conserved asparagine 297 (N297) glycosylation site in each of the $2 \mathrm{CH} 2$ domains. The glycans are buried within the hydrophobic core between the 2 heavy chains and influence Fc structure $(4,5)$. The biantennary core glycan structure, which is composed

Conflict of interest: The authors have declared that no conflict of interest exists. Submitted: May 18, 2015; Accepted: August 25, 2015.

Reference information: J Clin Invest. 2015;125(11):4160-4170. doi:10.1172/JCI82695. of $2 \mathrm{~N}$-acetylglucosamines (GlcNAc) and 3 mannose residues, can be further decorated with fucose, bisecting GlcNAc and terminal GlcNAc, galactose, and sialic acid. Genetic or enzymatic removal of this sugar moiety results in a loss of both pro- and antiinflammatory activities of $\operatorname{IgG}(1,6)$.

Antiinflammatory activities of IgG have been associated with the presence of sialic acid, based on observations that patients with autoimmune diseases such as rheumatoid arthritis show decreased levels of IgG Fc sialylation (7-9) and the finding that the antiinflammatory activity of i.v. immunoglobulins (IVIG) in various murine models of antibody-mediated autoimmune diseases could be recapitulated using sialylated Fc fragments derived from IVIG or a human IgG1 recombinant antibody at a 30-fold lower dose than IVIG (10-12).

The antiinflammatory activity of sialylated $\operatorname{IgG} \mathrm{Fc}$ has been attributed to its ability to induce the production of IL-33 by myeloid regulatory cells upon binding to the lectin DC-specific ICAM-3-grabbing nonintegrin receptor DC-SIGN, which, in turn, induces expansion of IL-4-producing basophils that promote increased expression of the inhibitory Fc $\gamma$ receptor Fc $\gamma$ RIIB, thereby increasing the activation threshold of innate effector cells to immune complexes (13). More recently, it has been demonstrated that IgG Fc sialylation acts as a negative regulator of B cell proliferation independent of Fc $\gamma$ RIIB expression $(14,15)$. Here, we show that Fc sialylation inhibits immediate proinflammatory IgG effector functions through impairment of complement-mediated cytotoxicity. 
A

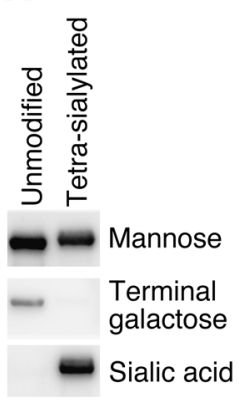

B Light chain

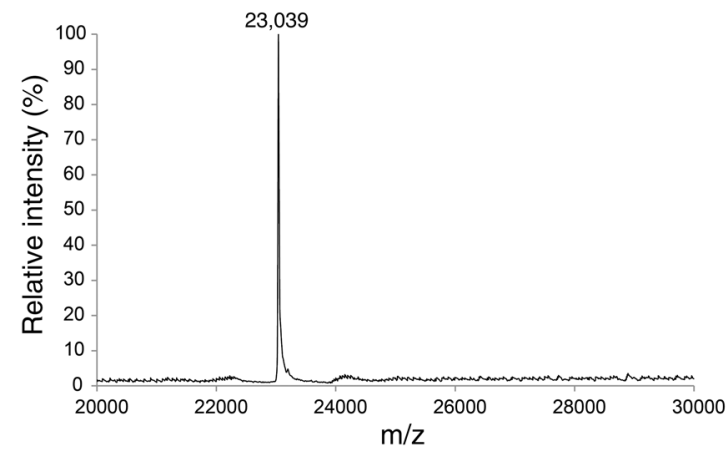

C Heavy chain

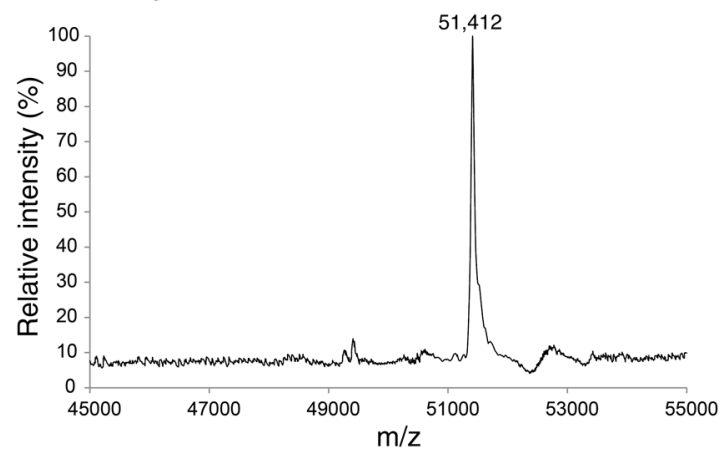

Figure 1. Characterization of unmodified and tetra-Fc-sialylated RTX. (A) Immunoblotting of RTX and tetra-Fc-sialylated RTX using lectins specific for individual glycans (mannose: LCA; terminal galactose: ECL; sialic acid: SNA). (B) Liquid chromatography-mass spectrometry (LC/MS) analysis of the tetraFc-sialylated RTX after reduction to separate the light chain and heavy chain.

\section{Results}

IgG Fc sialylation impairs complement-dependent cytotoxicity. Rituximab (RTX) is a chimeric mouse-human IgG1 monoclonal antibody that targets the CD20 antigen, which is expressed on B lymphocytes. RTX depletes B cells through a combination of antibody-dependent cell-mediated cytotoxicity (ADCC) and complement-dependent cytotoxicity (CDC) (16). The major Fc glycans of commercial RTX are core-fucosylated biantennary complex-type oligosaccharides carrying 0-2 galactose moieties buy lacking sialic acid (refs. 17, 18, and Figure 1A). A homogeneous, tetra-Fcsialylated glycoform (G2SA2) of RTX - i.e., carrying 4 sialic acids per Fc fragment (2 sialic acids per Fc glycan [Figure 1A and Supplemental Figure 1; supplemental material available online with this article; doi:10.1172/JCI82695DS1]) - was prepared by the chemoenzymatic glycosylation remodeling of commercial RTX (17), and the purity and identity of the fully sialylated RTX were confirmed by electron spray ionization-liquid chromatography/mass spectrometry (ESI-LC/MS) analysis of the light chain and heavy chain of the product (Figure 1B). We employed these 2 glycoforms-i.e., commercial RTX not carrying sialic acid versus tetra-Fc-sialylated RTX - to determine whether IgG Fc sialylation impacts the efficacy of CD20-targeted B cell depletion.

Incubation of natural killer cells (NK cells) with either the unmodified or tetra-Fc-sialylated antibody resulted in efficient lysis of autologous B cells, and the depleting efficacy was similar between the 2 glycoforms (Figure 2A). In addition, both glycoforms exhibited similar binding affinities for the activating $\mathrm{Fc}$ receptors Fc $\gamma$ RIIIA-158F and Fc $\gamma$ RIIIA-158V, which, upon ligation, mediate ADCC (Figure 2B). Binding affinities of both glycoforms were also similar for Fc $\gamma$ RI and Fc $\gamma$ RIIA (Supplemental Figure 1). In stark contrast, tetra-Fc-sialylated RTX was less efficient in inducing CDC in CD20 ${ }^{+}$Burkitt's lymphoma Raji and Ramos cells (Figure 2C). An alternative approach to achieve Fc sialylation is to react an Fc glycan containing substrate molecule with $\beta 1,4$ galactosyltransferase-1 $(\beta 1,4 \mathrm{GalT})$ and $\alpha 2,6$-sialyltransferase (ST6Gal1) enzymes in the presence of the corresponding sugar nucleotide substrates, which results in enrichment of mono- and bisialylated species (Supplemental Figure 2 and refs. 11, 19, 20). As shown in Figure 2D, enzymatic enrichment of RTX for sialylated glycoforms also resulted in decreased efficacy to induce CDC.
To determine whether the effect of Fc sialylation on CDC is RTX specific, we additionally enriched a monoclonal humanized IgG1 antibody recognizing myelin oligodendrocyte glycoprotein (MOG) and lacking Fc sialylation (hu8-18C5) (21) for sialylated species by $\beta 1,4 \mathrm{GalT}$ and ST6Gal1 treatment. CDC was assessed with an oligodendroglial cell line (MO3.13) transduced to express human full-length MOG (Figure 3 and ref. 22). Similar to RTX, sialylated glycoforms of the MOG-specific hu8-18C5 antibody were less efficient in inducing CDC compared with their nonsialic acid-carrying counterparts (Figure 2D). The impaired efficacy of sialylated IgG to induce CDC was not reflected by decreased antigen-binding affinities (Figure 3, C and D). These data indicate that IgG Fc sialylation impairs CDC independently from Fc receptor binding and antigen specificity.

$\mathrm{Fc}$ sialylation neutralizes increased $\mathrm{C1q}$ binding of Fc-galactosylated IgG. The classical complement pathway is triggered by binding of $\mathrm{C} 1 \mathrm{q}$ to the $\mathrm{Fc}$ domain in antibody/antigen complexes, leading to autoactivation of the $\mathrm{C} 1$ complex (comprising C1q, C1r, and C1s) and activation of complement by cleavage of $\mathrm{C} 4$ and $\mathrm{C} 2$. The resulting C4bC2a convertase cleaves $\mathrm{C} 3$, causing the covalent coupling of $\mathrm{C} 3 \mathrm{~b}$ to reactive surfaces such as the cell membrane, which culminates in the formation of the membrane attack complex and lysis of the target cell (23).

The binding affinity of $\mathrm{C1q}$ for murine IgG has been shown to be reduced upon the removal of galactose from the IgG Fc-linked glycan (24). To investigate the mechanism by which Fc sialylation impacts CDC, we first determined binding affinities and kinetics of unmodified and tetra-Fc-sialylated RTX with C1q by surface plasmon resonance (SPR) analysis. Compared with unmodified IgG, the rate of complex formation was lower for tetra-Fc-sialylated IgG $\left(\mathrm{k}_{\text {on }}=1.6\right.$ compared with $\left.3.9 / 10^{7}[\mathrm{~ms}]^{-1}\right)$ while the rate of dissociation was higher $\left(\mathrm{k}_{\text {off }}=0.22\right.$ compared with $\left.0.15 / \mathrm{s}^{-1}\right)$, resulting in a 3.7-fold increased $\mathrm{k}_{\mathrm{D}}$ for tetra-Fc-sialylated IgG $\left(\mathrm{k}_{\mathrm{D} \text { C1q:tetra-Fc-sialylated IgG }}=14\right.$; $\mathrm{k}_{\mathrm{DC1} \text { (qunmodified IgG }}=3.8$ ) (Figure 4). Next, we generated galactosylated, galactosylated and sialylated, and degalactosylated Fc glycovariants of RTX and hu8-18C5 (Figure 5, A and D, and Supplemental Figure 2) and tested them for their ability to induce cell-associated complement activation. Both antibodies contain the Fc-glycosylation site at N297 but lack additional fragment antigen binding (Fab) glycosylation sites (Supplemental Figure 3). 


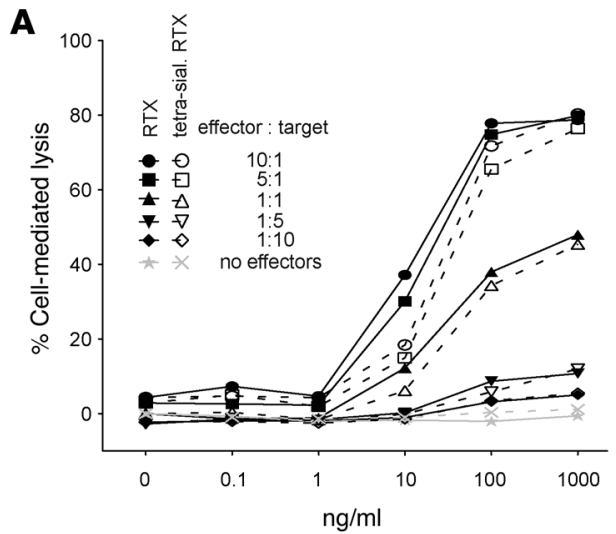

B
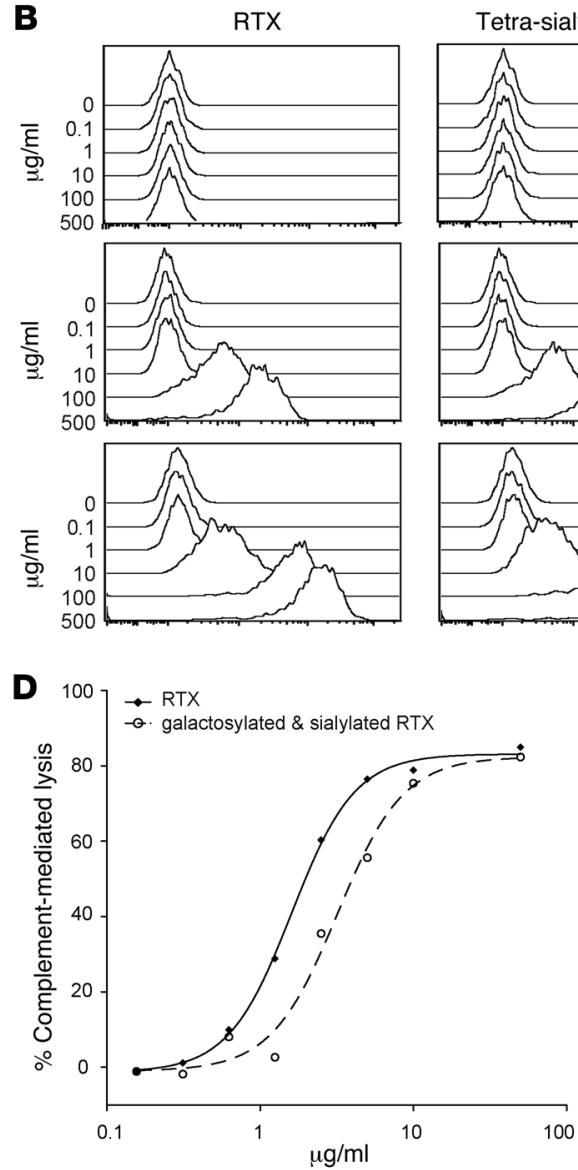

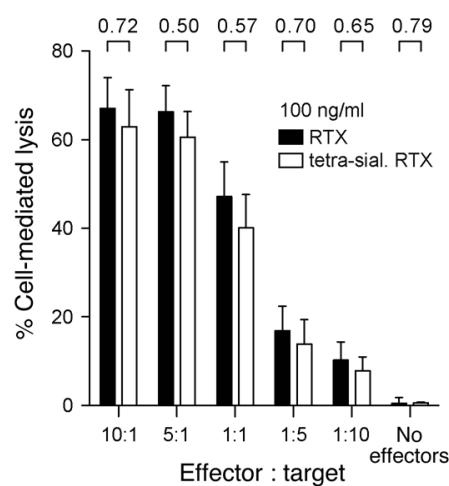

C

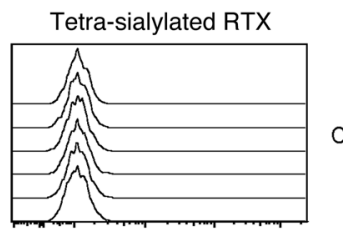

$\mathrm{CHO}$

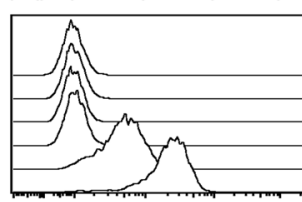

$\mathrm{CHO}$

FcyRIIla

(158F)

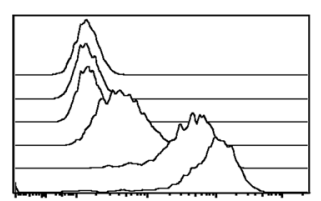

$\mathrm{CHO}$

FçRIIla

(158V)
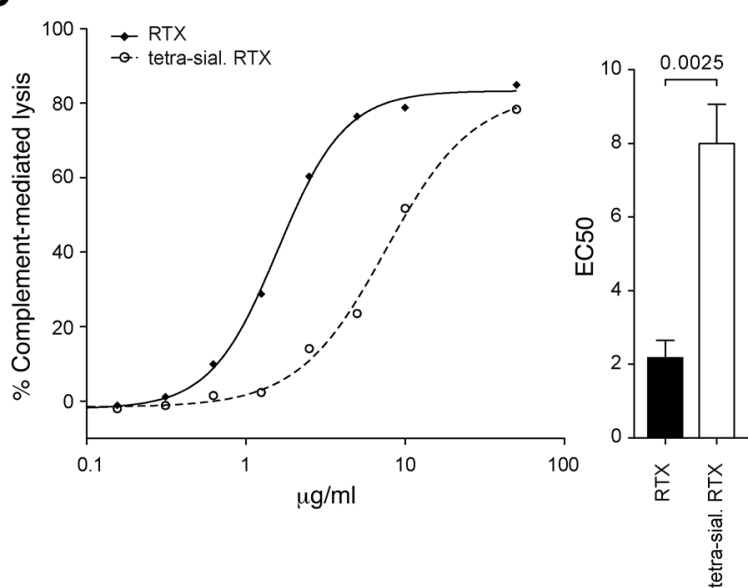

Figure 2. IgG Fc sialylation impairs complement-dependent but not cell-mediated cytotoxicity. (A) RTX and tetra-Fc-sialylated RTX mediated lysis of B cells (targets) by autologous NK cells (effectors). Representative experiment and quantification of 3 independent experiments. Statistics were performed by $t$ test. (B) Binding of RTX and tetra-Fc-sialylated RTX to CHO cells either untransfected or transfected with the low-affinity (158F) or high-affinity (158V) variant of Fc $\gamma R$ IIIIA. (C) Complement-mediated lysis of the CD20+ Raji cells. Representative experiment and quantification of EC50 of 3 independent experiments. Statistics were performed by $t$ test. (D) Complement-mediated lysis of CD20+ Raji cells (left) or MO3.13 MOG cells (right) with RTX and anti-MOC (hu8-18C5) or Fc-galactosylated and sialylated variants of the antibodies, representative of 3 independent experiments. 158F, Phenylalanine 158; 158V, Valine 158.

Incubation of target cells expressing the cell-surface antigens for RTX or hu8-18C5 in the presence of human serum complement led to rapid binding of $\mathrm{C} 1 \mathrm{q}$ (Figure 5). Fc galactosylation of unmodified antibodies resulted in a markedly increased capacity to bind C1q. Subsequent addition of sialic acid abrogated increased C1q binding of Fc-galactosylated IgG. Fully tetra-Fc-sialylated RTX was largely devoid of C1q binding (Figure 5). The capacity of Fc sialylation to block $\mathrm{C} 1 \mathrm{q}$ binding translated into decreased levels of C3b deposition to the cell surface (Supplemental Figure 4).

To investigate whether these effects can be transferred to polyclonal IgG, we purified sialylated Fc from IVIG and obtained desialylated $\mathrm{Fc}$ (i.e., terminally galactosylated $\mathrm{Fc}$ ) following neuraminidase treatment (Figure 6A). Similar to the monoclonal IgGs tested, enrichment of terminally galactosylated Fc resulted in 
A
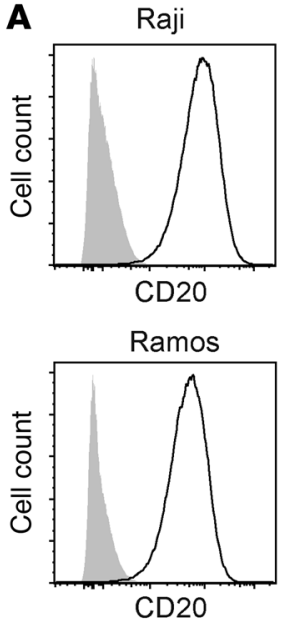

B $\mathrm{MO} .13 \mathrm{MOG}$

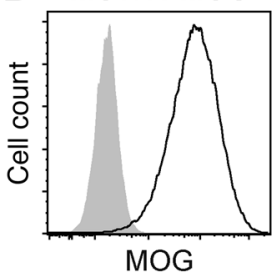

C

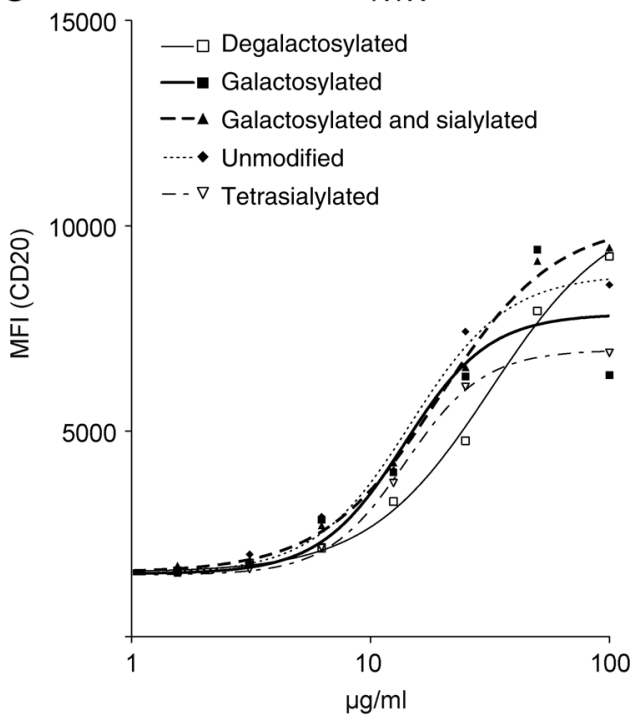

D

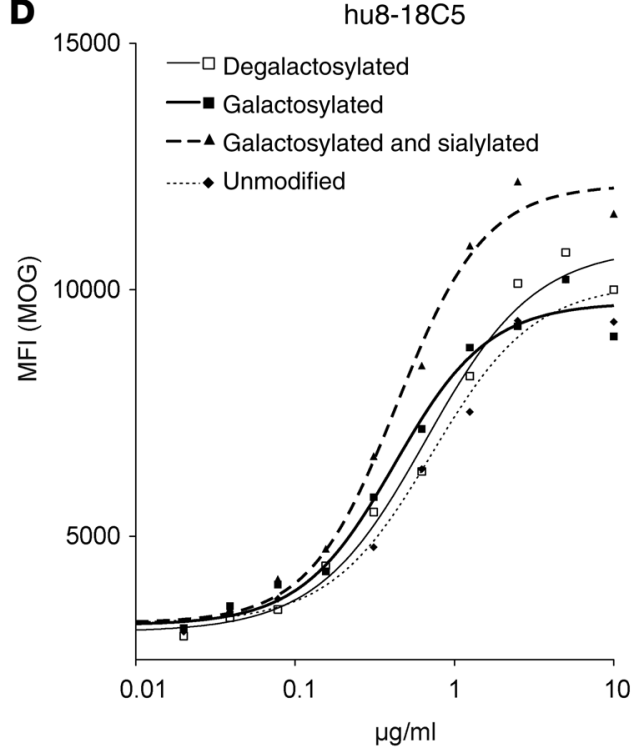

Figure 3. Cell-surface antigen expression on target cells and antigen binding properties of antibody glycovariants. (A) Raji and Ramos cells were analyzed for CD20 expression by flow cytometry. Gray histograms, unstained cells; open histograms, stained cells. (B) M03.13 cells lentivirally transduced to express human MOG were analyzed for MOG expression by flow cytometry. Gray histograms, unstained cells; open histograms, stained cells. CD20 Raji cells were incubated with RTX or glycovariants of RTX (C) and M03.13 MOG cells with hu8-18C5 (anti-MOG) or glycovariants of hu8-18C5 (D) following incubation with a fluorescently labeled anti-Ig antibody and detection of antibody binding by flow cytometry. MFI, median fluorescence intensity.

increased binding of $\mathrm{C} 1 \mathrm{q}$, and the presence of sialic acid abrogated increased C1q binding of galactosylated Fc fragments (Figure 6B). These data indicate that Fc sialylation exerts immunoregulatory functions through inhibition of $\mathrm{C1q}$ binding and activation of the classical complement pathway, leading to reduced complementmediated cytotoxicity.

IgG Fc sialylation is associated with autoimmune disease remission. To address the clinical significance of our findings, we investigated whether IgG Fc sialylation is associated with disease activity in patients with chronic inflammatory demyelinating polyneuropathy (CIDP), the most common chronic autoimmune neuropathy (25). Humoral immune responses are considered to have a crucial role in mediating peripheral nerve damage in $\operatorname{CIDP}(26,27)$. Complement component $3 \mathrm{~d}$ is deposited on the outer surface of Schwann cells and the compact myelin in biopsies from patients with $\operatorname{CIDP}(28,29)$. Sera and IgG antibodies from CIDP patients induce peripheral demyelination in host animals (30), can increase the permeability of the bloodnerve barrier, and impair nerve conduction in various models of peripheral neuropathies (27). Moreover, removal of humoral immune mediators by plasma exchange is associated with short-term clinical disease remission and first-line therapy in $\operatorname{CIDP}(31,32)$.

First, we analyzed 19 patients with CIDP who were included in a randomized placebo-controlled clinical trial testing the efficacy of i.v. injection of $10 \%$ caprylate-chromatography-purified IVIG $-2 \mathrm{~g} /$ $\mathrm{kg}$ body weight initially and $1 \mathrm{~g} / \mathrm{kg}$ body weight every 3 weeks thereafter for 24 weeks - compared with $0.1 \%$ albumin (placebo) (32). In this trial, disease remission was defined as improvement in clinical disease severity after 24 weeks and was observed in 32 of 59 (54\%) patients treated with IVIG and 12 of 58 (21\%) patients who received placebo (32). We found that patients with clinical disease remission during placebo therapy could be distinguished from those with stable or worsened disease by the induction of Fc sialylation (Figure 7A). Changes in Fc galactosylation and fucosylation were similar in patients with disease remission compared with stable or worsened disease. In patients who received IVIG, we detected an increase of total IgG serum levels 2 weeks after the last infusion compared with baseline levels (Supplemental Figure 5A), which precluded profiling of endogenous IgG in this cohort. We therefore analyzed serum samples from an independent, additional cohort of 33 patients (termed Marburg cohort) in whom posttreatment samples were taken 3-5 weeks following the last infusion of IVIG (mean \pm SD: $4 \pm 0.6$ ) and in which total serum IgG levels after IVIG therapy were unchanged compared with pretreatment samples (Supplemental Figure 5B). In this cohort, reduction in clinical disease severity scores upon IVIG therapy was significantly associated with an induction of IgG Fc sialylation ( $r=0.63, P=0.0001$ ) (Figure $7 \mathrm{~B})$. We consider it unlikely that we profiled the sialic acid content of the IVIG product used to treat these patients, since the correlation of Inflammatory Neuropathy Cause and Treatment (INCAT) disease score reduction with increased $\operatorname{IgG}$ Fc sialylation remained significant after exclusion of 3 patients who showed substantially increased IgG levels $3-5$ weeks after IVIG therapy as compared with pretreatment levels $(r=0.57$, 

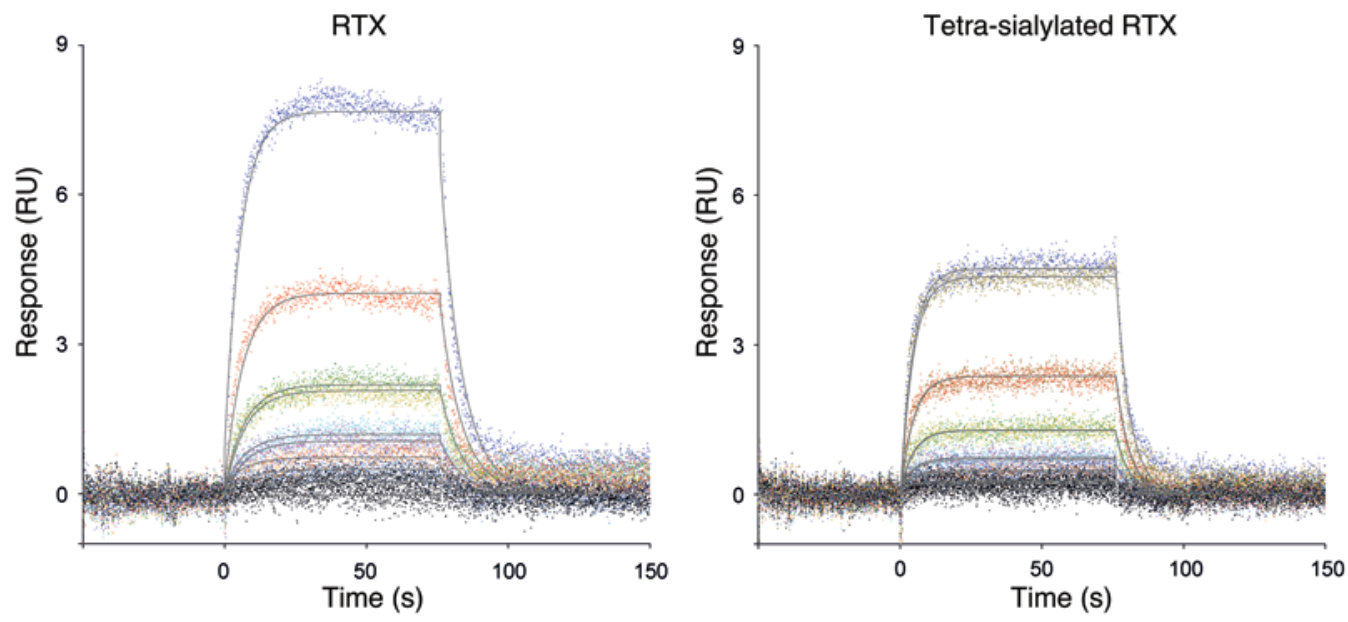

\begin{tabular}{|c|c|c|c|c|c|}
\hline Antibody & $\begin{array}{c}\text { C1q } \\
\text { Conc./ } / \mathrm{nM}\end{array}$ & $\begin{array}{c}\mathrm{k}_{\mathrm{on}^{1}}{ }^{1 /} \\
10^{7}(\mathrm{~ms})^{-1}\end{array}$ & $\begin{array}{c}\mathrm{k}_{\text {off }}{ }^{1 /} \\
\mathrm{s}^{-1}\end{array}$ & $\begin{array}{c}\mathrm{kD} / \\
\mathrm{nM}\end{array}$ & $\chi^{2}$ \\
\hline RTX & $0.02-1.3$ & $3.87 \pm 0.10$ & $0.146 \pm 0.002$ & $3.8 \pm 0.1$ & 0.0471 \\
\hline Tetra-sialylated RTX & $0.02-1.3$ & $1.59 \pm 0.06$ & $0.219 \pm 0.002$ & $14 \pm 0.5$ & 0.0929 \\
\hline
\end{tabular}

Figure 4. SPR of C1q binding to RTX and tetra-Fc-sialylated RTX. Sensorgrams and results of global fits (table) from SPR measurements.

$P=0.0011)$. Moreover, Fc-sialylated glycoforms represent only a minor fraction of glycovariants present in IVIG products (6), and the increase of total IgG serum levels 2 weeks after the last infusion in the first cohort of patients in remission was not associated with an upregulation of Fc sialylation (Supplemental Figure 5C). No significant correlation was observed for IgG Fc galactosylation, fucosylation, and the reduction of disease severity (Figure 7B).

We additionally determined complement activation in patients with CIDP from whom additional serum samples were available (Marburg cohort). Levels of soluble terminal complement complex (TCC, SC5b-9) generated by the assembly of C5b through C9 and previously shown to correlate with disease activity in patients with SLE (33) were quantified by ELISA according to the manufacturer's recommendation. CIDP patients with high levels of Fc-sialic acid induction - as defined by at least 2-fold induction of Fc sialylation - showed significantly lower levels of complement activation following IVIG therapy compared with baseline levels (Figure 7C). Thus, induction of Fc sialylation is associated with both spontaneous and treatment-induced disease remission, as well as decreased levels of TCC formation.

\section{Discussion}

Complement activation is a potent proinflammatory mechanism by which IgG antibodies trigger immune responses during infection, and its deregulation causes tissue damage in a wide array of human inflammatory, degenerative, and autoimmune diseases (23). Our study shows that the antiinflammatory activities of sialylated IgG Fc include the impairment of complement-dependent cytotoxicity by reducing the binding affinity of IgG Fc to C1q. Induction of $\operatorname{IgG} \mathrm{Fc}$ sialylation is associated with clinical disease remission in CIDP, an autoimmune disease in which humoral immune responses are central in mediating tissue damage.
We profiled total serum IgG Fc glycovariants since no specific pathogenic autoantibody or single triggering antigen has yet been identified in CIDP (34). Thus, while our data do not demonstrate induction of sialylation in specific autoantibodies causing the disease, they indicate that induction of Fc sialylation in CIDP sera is associated with clinical disease remission.

The observed mechanism of action of Fc sialylation was not identified in animal models of autoimmune diseases in which IVIG therapy and Fc sialylation proved to be effective, such as the $\mathrm{K} / \mathrm{BxN}$ serum transfer arthritis model and the antiplatelet monoclonal antibody 6A6-mediated model for immune thrombocytopenia $(10,11,13,20)$. However, the aforementioned autoimmune disease models are largely mediated by IgG-dependent activation of innate immune effector cells via crosslinking of cellular Fc $\gamma$ Rs, whereas IgG-dependent activation of the classical complement pathway via C1q was shown to be largely dispensable for disease development $(1,24,35-49)$.

The N-glycan resides in the $\mathrm{CH} 2$ domain, which is required for C1q binding (50). For human IgG1, 4 spatially close sites on the surface of the antibody - D270, K322, P329, and P331 - were shown to constitute the C1q binding epicenter (51). However, most of the aforementioned sites are conserved in human IgG isotypes that are deficient in C1q binding; it has therefore been suggested that the composition of the N-glycan might be critical for the antibodies' conformation and its ability to bind $\mathrm{C} 1 \mathrm{q}(51,52)$. In line with this assumption, we observed that presence of terminal galactose increases binding affinities for $\mathrm{C} 1 \mathrm{q}$ and that subsequent addition of sialic acid inhibited the increased C1q binding. Crystallographic and biophysical studies on sialylated and asialylated IgG Fc fragments have shown that IgG Fc sialylation disrupts glycan interactions with amino acid residues of the $\mathrm{CH} 2$ domain, leading to significant conformational changes seen in the protein structure 
A
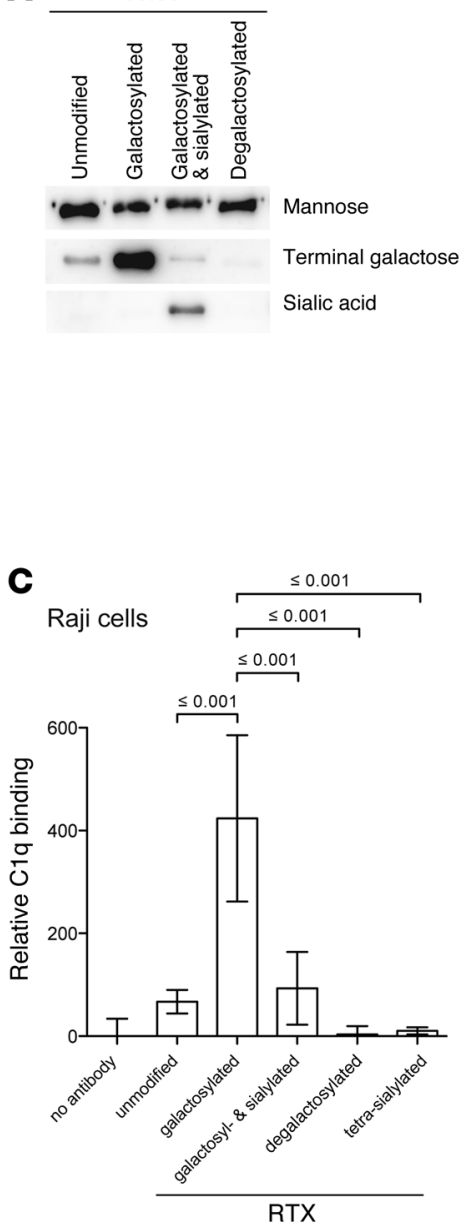

D

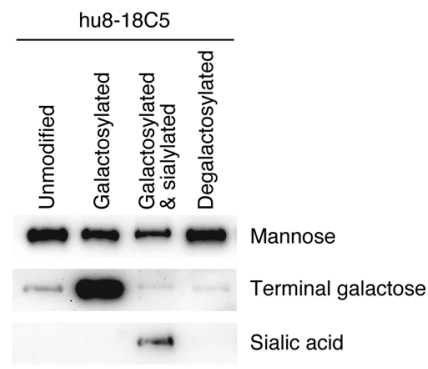

F MO3.13 MOG cells

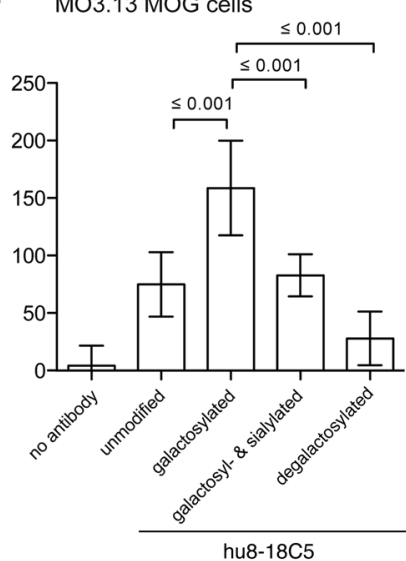

B
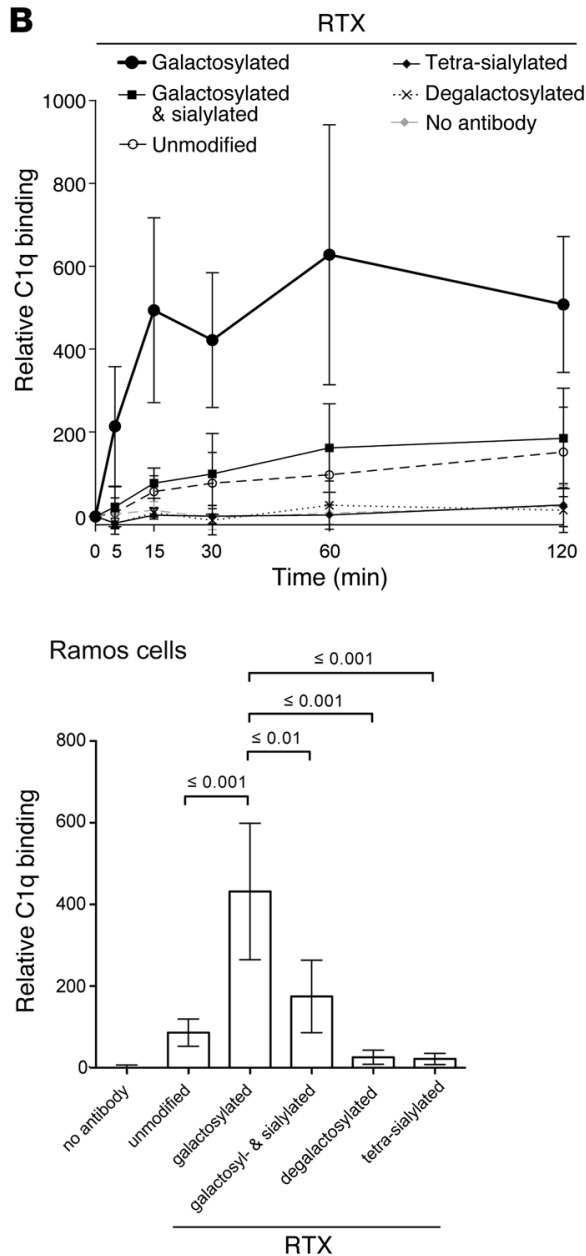

E

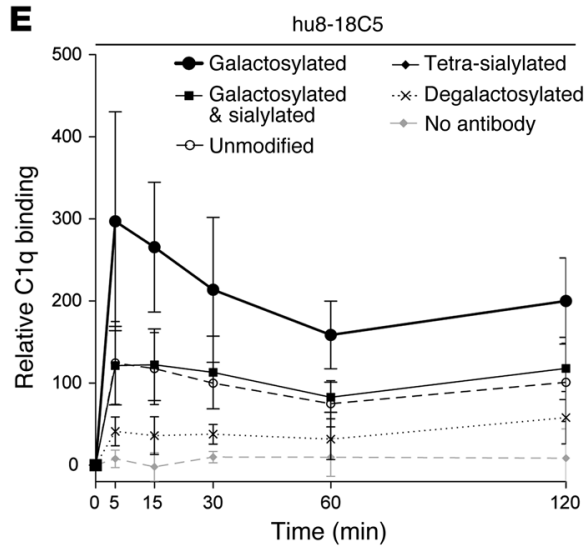

$\mathbf{G}$
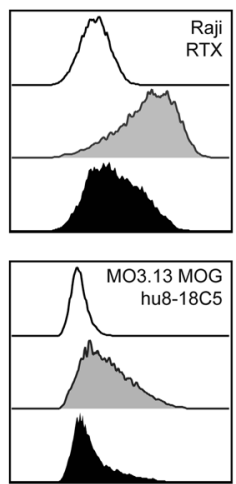

Figure 5. C1q binding to IgG Fc glycovariants.

(A) Lectin immunoblotting of RTX glycovariants. (mannose: LCA; terminal galactose: ECL; sialic acid: SNA). (B) Time-course of $\mathrm{C} 1 \mathrm{q}$ binding to $\mathrm{CD} 2 \mathrm{O}^{+}$Raji cells in the presence or absence of differentially glycosylated RTX. Mean \pm SD of 6 independent experiments. Median fluorescence intensity obtained for the unmodified antibody after 60 minutes was set to 100 , and the relative signals were calculated. (C) Quantification of RTX antibody glycovariant-dependent $\mathrm{C} 1 \mathrm{q}$ binding to $\mathrm{CD} 2 \mathrm{O}^{+}$Raji and Ramos cells after 30 minutes incubation. Mean \pm SD of at least 3 independent experiments. Statistics were performed by 1-way ANOVA and Bonferroni post test. (D) Lectin immunoblotting of hu8-18C5 (anti-MOG) glycovariants. (E) Timecourse of $\mathrm{C} 1 \mathrm{q}$ binding to M03.13 MOC cells in the presence or absence of differentially glycosylated variants of hu8-18C5. Mean \pm SD of 8 independent experiments. Median fluorescence intensity obtained for the unmodified antibody after 30 minutes was set to 100 and the relative signals were calculated. (F) Quantification of hu8-18C5 antibody glycovariant-dependent $\mathrm{C} 1 \mathrm{q}$ binding to M03.13 MOG cells after 60 minutes. Statistics were performed by 1-way ANOVA and Bonferroni post test. (C) Representative flow cytometry stainings showing $\mathrm{C} 1 \mathrm{q}$ binding to the indicated cell type and antibody after 1 hour incubation.

$(53,54)$. These data suggest that addition of sialic acid induces structural changes in IgG $\mathrm{Fc}$ fragments, which translate into reduced C1q binding and CDC.

Taken together, we identified an Fc $\gamma$ Rindependent mechanism by which $\mathrm{Fc}$ sialylated glycovariants acquire antiinflammatory properties and limit proinflammatory $\operatorname{IgG}$ effector function. This mechanism might complement immunomodulatory effects of sialylated IgG associated with upregulation of the inhibitory FcyRIIB $(1,55)$ and supports the development of strategies that target increased Fc sialylation for the treatment of human autoimmune diseases (56).

\section{Methods}

Patients. Samples and clinical data were collected during a randomized placebo-controlled trial (ICE trial) testing the efficacy of i.v. injection of $10 \%$ caprylate-chromatography purified immunoglobulin (IGIV-C, Gamunex, Grifols Therapeutics Inc.) in patients with CIDP (32). Patients included in the ICE trial did not receive IVIG treatment in the 3 months before study entry. An additional cohort of patients was recruited between 2010 and 2013 at the Department of Neurology, University of Marburg, Germany (Supplemental Table 1). All patients fulfilled the European Federation of Neurological Societies/Peripheral Nerve Soci- 
A

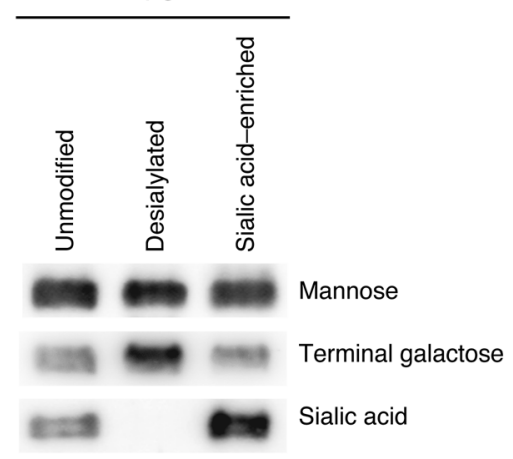

B

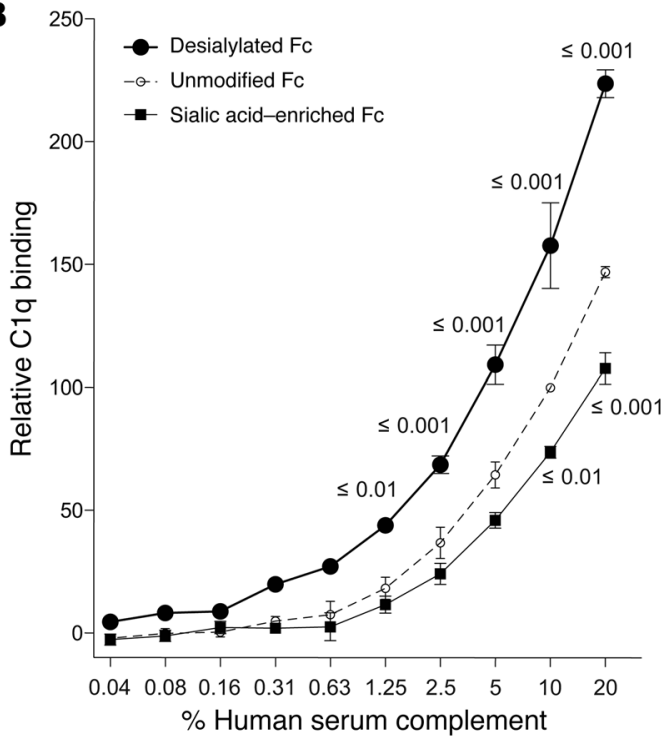

Figure 6. C1q binding to human polyclonal IgG Fc glycovariants. (A) Lectin immunoblotting of Fc purified from human polyclonal IgG and enriched for sialylated Fc or desialylated (mannose: LCA; terminal galactose: ECL; sialic acid: SNA). (B) C1q binding to polyclonal IgC Fc glycovariants measured by ELISA. Mean and \pm SEM of 3 independent experiments. C1q binding was normalized to unmodified Fc at $10 \%$ serum. Individual curves were compared with Fc using 2-way ANOVA and Bonferroni post test comparing $\mathrm{Fc}$ and desialylated $\mathrm{Fc}$, or Fc and sialic acid-enriched Fc ( $P$ values below curve). ety diagnostic criteria for CIDP. They were prospectively followed during IVIG therapy using the INCAT score to monitor clinical treatment response. Patients did not receive IVIG therapy before study entry. Serum samples were collected at each visit.

Buffers and reagents. RPMI-1640, DMEM, penicillin-streptomycin (P/S), pooled human serum, TO-PRO-3 Stain, LIVE/DEAD Fixable Near-IR Dead Cell Stain Kit, LIVE/DEAD Fixable Aqua Dead Cell Stain Kit, 1-Step Ultra TMB-ELISA Substrate Solution and CaptureSelect IgG-CH1 PRO Affinity Matrix were purchased from Invitrogen. Recombinant human IL-2 was purchased from PeproTech. PKH26 Red Fluorescent Cell Linker Kit was purchased from Sigma-Aldrich. Ficoll-Paque was purchased from GE Healthcare. Accutase was purchased from StemCell Technologies Inc. MACS NK Cell Isolation Kit-II (negative selection) and B Cell Isolation Kit (CD19+ ${ }^{+}$selection) were purchased from Miltenyi Biotec. Human serum complement, C5 depleted human serum, MicroVue SC5b-9 Plus ELISA, and recombinant human C1q were purchased from TECOmedical Group. EZ-Link NHS-PEG12-Biotin was purchased from Thermo Scientific. Papain and Nutridoma-SP were purchased from Roche Applied Science. HiLoad 16/60 Superdex 200 column, HiTrap Protein G HP Columns, StrepTrap HP Columns, HisTrap HP columns, and Protein G Sepharose 4 Fast Flow were purchased from GE Healthcare. Ceramic Fluoroapatite (CFT-II, $40 \mu \mathrm{M}$ ) was purchased from Bio-Rad. Biotinylated lectins (Lens culinaris agglutinin [LCA], Sambucus nigra agglutinin [SNA], Erythrina cristagalli lectin [ECL], and Aleuria aurantia lectin [AAL]) and SNA-Agarose were purchased from Vector Laboratories. Neuraminidase was purchased from New England Biolabs Inc. UDP-Galactose, CMP-Sialic Acid, $\beta 1,4-$ Galactosidase were purchased from Merck. StarGate cloning and expression system was purchased from IBA. CMD500L sensor chip was from Xantec.

Antibodies and streptavidin. RTX (MabThera, catalog 10129436) was purchased from Roche Applied Science. Monoclonal mouse anti-human C1q (catalog A201), biotinylated anti-human C1q (cata$\log$ A700), and anti-human-C3c (catalog A701) were purchased from TECOmedical Group. Anti-human IgG Fc-HRP (catalog A0170) was purchased from Sigma-Aldrich. Anti-CD20-PE-Cy7 (clone 2H7) was purchased from eBioscience. Polyclonal rabbit anti-mouse IgG $(\mathrm{H}+\mathrm{L})$ Alexa Fluor 488 (catalog A-11059) was purchased from Invitrogen. Streptavidin-FITC (catalog 405202) and streptavidin-PE (catalog 405204) were purchased from BioLegend.

CDC assay. Raji or Ramos cells expressing the target antigen of RTX (CD20; Figure 3A) were used to assess RTX-mediated CDC. Therefore, $7 \times 10^{4}$ cells were placed in a humidified incubator at $37^{\circ} \mathrm{C}$ and $5 \% \mathrm{CO}_{2}$ in $\mathrm{RPMI}-1640$ containing $\mathrm{P} / \mathrm{S}(50 \mathrm{U} / \mathrm{ml})$ in 96-well V-bottom plates in the presence or absence of RTX or glycovariants of it. After 30 minutes, human serum complement was added to a final concentration of $5 \%$, and the incubation was continued for 12 hours. For hu8-18C5-mediated CDC, $2 \times 10^{4}$ MO3.13 MOG cells were seeded in 96-well flat-bottom plates in DMEM plus $10 \%$ FCS. On the next day, the medium was removed, and DMEM containing $15 \%$ serum complement with or without hu8-18C5 or a glycovariant of it was added following incubation for 4 hours in a humidified incubator at $37^{\circ} \mathrm{C}$ and $5 \% \mathrm{CO}_{2}$. For cell-death assessment, TO-PRO-3 stain was added to a final concentration of 200 nM. Cells were acquired using BD FACS Canto-II (BD Biosciences) and analyzed with FlowJo (Tree Star).

ADCC assay. ADCC assay was performed as previously described (57). Human peripheral blood mononuclear cells (PBMC) were isolated from buffy coats using Ficoll-Paque. NK cells were negatively selected using MACS NK cell isolation kit, and B cells were positively selected using MACS CD19+ selection kit. Both cells were cultured overnight in RPMI supplemented with $\mathrm{P} / \mathrm{S}(50 \mathrm{U} / \mathrm{ml})$ and $5 \%$ heatinactivated pooled human serum in 24 -well plates at $5 \times 10^{6}$ cells per well. NK cell medium was additionally supplemented with $100 \mathrm{IU}$ IL-2. On the next day, B cells were stained with PKH26 according to the manufacturer's recommendation. B cells $\left(1 \times 10^{4}\right)$ were incubated in the presence of autologous NK cells $\left(1 \times 10^{3}-1 \times 10^{5}\right)$ and RTX $(0.1$ $\mu \mathrm{g} / \mathrm{ml}-1 \mathrm{ng} / \mathrm{ml})$ in RPMI supplemented with P/S (50 U/ml), $5 \%$ heatinactivated pooled human serum, and 100 IU IL-2 in 96-well roundbottom plates for 18 hours in a humidified incubator at $37^{\circ} \mathrm{C}$ and $5 \%$ $\mathrm{CO}_{2}$. For cell-death assessment, TO-PRO-3 stain was added to a final concentration of $200 \mathrm{nM}$. Cells were acquired using BD FACS Canto-II and analyzed with FlowJo. 


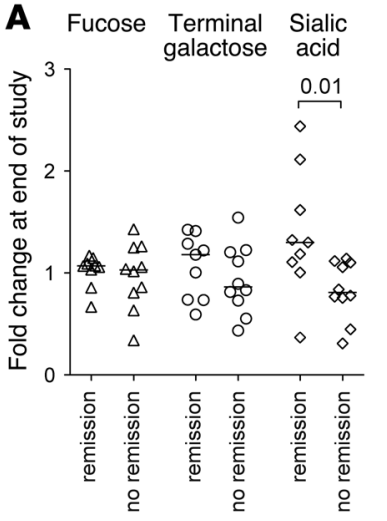

B

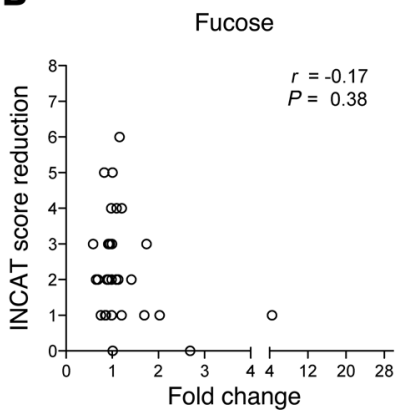

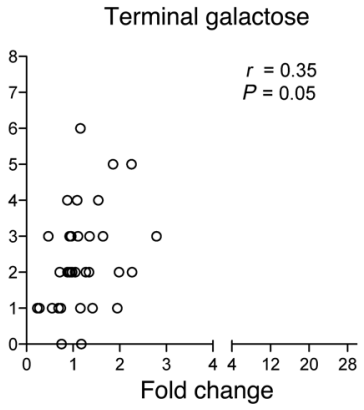

C

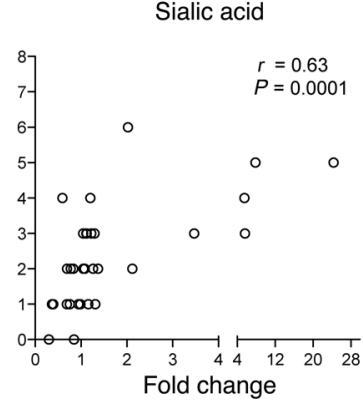

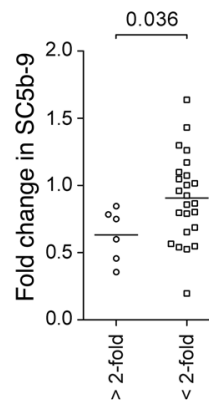

Sialic acid induction

Figure 7. Induction of IgC Fc sialylation is associated with disease remission in CIDP. (A) Serum IgC Fc glycan composition of CIDP patients analyzed by lectin blotting. Relative IgG Fc glycan abundance of CIDP patients before and after a 24-week observation period. Patients undergoing spontaneous disease remission are compared with those with stable or worsening disease (no remission). Statistics were performed by Mann-Whitney $U$ test. (B) IgG Fc glycan composition analysis of CIDP patients (Marburg patient cohort) before and 3-5 weeks after receiving the last IVIC injection. Fold changes in glycan composition are compared with disease activity changes reflected by the INCAT score. Statistics were performed by Spearman test. (C) Complement activation as defined by serum levels of the TCC (SC5b-9) quantified by ELISA before and 3-5 weeks after IVIC therapy (Marburg patient cohort). Statistics were performed by Mann-Whitney $U$ test.

Fc $R$ R binding assay. RTX and tetra-Fc-sialylated RTX were biotinylated using EZ-Link NHS-PEG12-Biotin according to the manufacturer's recommendations. CHO cells $\left(1 \times 10^{5}\right)$ stably expressing none or 1 of the human Fc $\gamma$ Rs (58), a gift from Falk Nimmerjahn (Department of Biology, University of Erlangen-Nuremberg, Germany), were incubated for 30 minutes on ice in the presence of RTX or tetra-Fcsialylated RTX. Cells were washed twice with PBS and incubated for 30 minutes with streptavidin-FITC for detection of binding and LIVE/ DEAD Fixable Near-IR Dead Cell Stain Kit for dead cell exclusion. After washing twice with PBS, cells were acquired using BD FACS Canto-II and analyzed with FlowJo.

Purification of $F c$, desialylated $F c$, and sialic acid-enriched $F c$ from polyclonal IgG. IVIG (Pivigen, CSL Behring) was used as source of human IgG. IgG (550 mg) was incubated in $0.02 \mathrm{M}$ EDTA and 0.02 $\mathrm{M} \mathrm{L}$-cysteine in PBS at pH 6.5 with $0.8 \mathrm{mg}$ papain for 2 hours at $25^{\circ} \mathrm{C}$. Uncut IgG and papain were separated by size exclusion with a HiLoad 16/60 Superdex 200 column and GE Aekta purifier. The fraction containing the $\mathrm{Fc}$ and $\mathrm{Fab}$ fragments was concentrated and dialyzed to $5 \mathrm{mM}$ Na-phosphate buffer $\mathrm{pH}$ 7.1. Fab and Fc were separated using a Ceramic Fluoroapatite column (CFT-II, $40 \mu \mathrm{m}$ ), which binds Fab but not Fc. For purification of sialic acid-enriched Fc, Fc was dialyzed to TBS and $\mathrm{CaCl}_{2}$ was added to a final concentration of $0.1 \mathrm{M}$. Sialic acid-enriched Fc was purified from $40 \mathrm{mg} \mathrm{Fc}$ by binding to $4 \mathrm{ml} \mathrm{SNA-}$ Agarose and elution with $0.5 \mathrm{M}$ lactose in TBS as previously described (11). For neuraminidase treatment, Fc was dialyzed to $50 \mathrm{mM}$ sodium citrate $\mathrm{pH} 6$ and incubated with 1 unit neuraminidase per $\mu \mathrm{g}$ Fc for 48 hours at $37^{\circ} \mathrm{C}$. Neuraminidase was removed using Protein-G sepharose 4 Fast Flow. For further purification, Fc, desialylated Fc, and sialic acid-enriched Fc were cleared from residual Fab using a CaptureSelect IgG-CH1 pro affinity matrix and reapplied to a HiLoad 16/60 Superdex 200 column.

Purification of hu8-18C5 antibody. The hu8-18C5 antibody has been generated by cloning of the 8-18C5 hybridoma-derived Igh and $I g k$ variable-region sequences into human IgG1 heavy-chain and $\kappa$ light chain expression vectors as previously described (21).
hu8-18C5 was purified by cotransfecting the calcium phosphate precipitated expression vectors in HKB-11 cells. After 12 hours, the medium was exchanged to DMEM containing P/S (50 U/ml) and $1 \%$ Nutridoma-SP. The culture supernatant was harvested 4 days later, and antibodies were purified using HiTrap Protein G HP columns according to the manufacturer's instructions using GE Aekta prime plus.

Purification of recombinant glycosyltransferases. For purification of sialyl- and galactosyl-transferases, cDNA of bovine $\beta 1,4 \mathrm{GalT}$ and human ST6Gal1 was cloned into PCSG-IBA-144 expression vectors using the StarGate cloning system (IBA) according to the manufacturer's instructions. This yields plasmids encoding for proteins containing an N-terminal Twin-Strep-tag and a C-terminal Hexahistidinetag. $293 \mathrm{~T}$ cells were cultured under standard cell-culture conditions in DMEM containing 10\% FCS and P/S (50 U/ml) and transfected with calcium-phosphate precipitated plasmids encoding either $\beta 1,4 \mathrm{GalT}$ or ST6Gal1. After 12 hours, the medium was replaced with fresh DMEM containing $10 \% \mathrm{FCS}$ and $\mathrm{P} / \mathrm{S}(50 \mathrm{U} / \mathrm{ml})$, and cells were cultured for another 3 days. The cell supernatant was harvested, and the proteins were purified by immobilized metal ion affinity chromatography (HisTrap HP columns) and subsequently Strep-Tactin affinity chromatography (Strep-Trap HP columns) according to the manufacturer's instructions using GE Aekta prime plus.

Generation of antibody glycovariants. Tetra-Fc-sialylated RTX was generated by chemoenzymatic glycoengineering as previously described (17) with adequate modifications. Briefly, commercial RTX (8 mg) was incubated with EndoS WT $(25 \mu \mathrm{g})$ at $37^{\circ} \mathrm{C}$ for 1 hour, and the completion of deglycosylation was confirmed by LC/MS analysis. The deglycosylated antibody was purified using a HiTrap protein A column, and the eluted fractions were concentrated and buffer exchanged into $1 \times \mathrm{PBS} \mathrm{pH}$ 7.4. The purified Fuc- $\alpha 1,6-$ GlcNAc-RTX (7 mg) and sialylated complex type oxazoline $(3.5 \mathrm{mg})$ were incubated with EndoS D233Q $(175 \mu \mathrm{g})$ at $37^{\circ} \mathrm{C}$ in $100 \mathrm{mM}$ Tris $\mathrm{pH} 7.4$ (500 $\mu$ l total volume). The reaction progress was monitored using LC/MS analysis, and complete transfer was 
achieved in 1.5 hours. The tetra-Fc-sialylated RTX was purified using a HiTrap protein A column and was concentrated and buffer exchanged into $1 \times$ PBS pH 7.4. LC/MS: heavy chain of RTX with sialylated complex type glycan, $\mathrm{M}=51412 \mathrm{Da}$; found (m/z), 51412 (deconvolution data). Enzymatic galactosylation and sialylation were performed as previously described $(11,19)$. For galactosylation, antibodies were buffer-exchanged to $0.2 \mathrm{mM}$ MES pH 6.5 and incubated for 24 hours at $37^{\circ} \mathrm{C}$ in the presence of $5 \mu \mathrm{g} \beta 1,4 \mathrm{GalT}$ per mg antibody, $10 \mathrm{mM}$ UDP-galactose, and $20 \mathrm{mM} \mathrm{MnCl}_{2}$. Nongalactosylated antibodies (unmodified) were treated the same way but without the addition of UDP-galactose and $\beta 1,4 \mathrm{GalT}$. After buffer exchange to $25 \mathrm{mM}$ MOPS $100 \mathrm{mM} \mathrm{KCl}$ (pH 7.2), Fcgalactosylated antibodies were Fc-sialylated by the addition of 50 $\mu \mathrm{g}$ ST6Gal1 per mg antibody and CMP-sialic acid at a final concentration of $1.5 \mathrm{mM}$. The reaction was incubated for 24 hours at $37^{\circ} \mathrm{C}$. Nonsialylated antibodies (unmodified and galactosylated) were treated the same way but without the addition of CMP-sialic acid and ST6Gal1. All antibodies were buffer-exchanged to TBS. To enrich for antibodies containing sialic acid, SNA lectin affinity chromatography was performed as previously described (11). For degalactosylation, RTX or hu8-18C5 was dialyzed to $50 \mathrm{mM}$ sodium phosphate $\mathrm{pH} 6.0$ and incubated 6 hours at room temperature and 1 hour at $37^{\circ} \mathrm{C}$ in the presence of $30 \mathrm{mU} \beta 1,4$-galactosidase per $\mathrm{mg}$ antibody. Finally, all antibodies were purified using protein- $\mathrm{G}$ sepharose 4 fast flow according to the manufacturer's instruction, dialyzed to PBS, and sterilized by $0.2 \mu \mathrm{M}$ filtration. Acrylamide gel electrophoresis and silver staining were performed to test antibody integrity and purity, and lectin-blotting was used to confirm glycan modifications.

C1q binding assay. For C1q binding to Raji or Ramos cells, $2 \times 10^{5}$ cells were seeded in RPMI-1640 containing 1\% C5-depleted human serum in the presence of $10 \mu \mathrm{g} / \mathrm{ml}$ RTX or RTX glycovariants for $5,15,30,60$, or 120 minutes in a humidified incubator at $37^{\circ} \mathrm{C}$ and $5 \% \mathrm{CO}_{2}$. For $\mathrm{C} 1 \mathrm{q}$ binding to MO3.13 MOG cells, cells were detached using accutase, and $1 \times 10^{5}$ cells were seeded in DMEM containing $1 \%$ C5-depleted serum in the presence of $2.5 \mu \mathrm{g}$ hu8-18C5 or hu8$18 \mathrm{C} 5$ glycovariants for $5,15,30,60$, or 120 minutes in a humidified incubator at $37^{\circ} \mathrm{C}$ and $5 \% \mathrm{CO}_{2} . \mathrm{C} 1 \mathrm{q}$ binding was detected by staining on ice with biotinylated anti-C1q for 1 hour. Cells were washed twice with PBS and incubated on ice with streptavidin-PE and LIVE/ DEAD Fixable Aqua Dead Cell Stain Kit for 25 minutes. After washing twice with PBS, samples were acquired using BD FACS Canto-II and analyzed with FlowJo.

C3b deposition assay. Raji cells $\left(2 \times 10^{5}\right)$ were seeded in RPMI-1640 containing 1\% C5-depleted serum in the presence of $10 \mu \mathrm{g} / \mathrm{ml} \mathrm{RTX}$ or RTX glycovariants for $5,15,30,60$, or 120 minutes in a humidified incubator at $37^{\circ} \mathrm{C}$ and $5 \% \mathrm{CO}_{2}$. For C3 deposition assessment, cells were stained with biotinylated anti-C3c for 1 hour on ice. Cells were washed twice with PBS and incubated on ice with streptavidin-PE and LIVE/DEAD Fixable Aqua Dead Cell Stain Kit for 25 minutes. After washing twice with PBS, samples were acquired using BD FACS Canto-II and analyzed with FlowJo.

C1q binding ELISA. Microtiter plates were coated overnight at $4^{\circ} \mathrm{C}$ with $0.5 \mu \mathrm{g} \mathrm{Fc}$, desialylated $\mathrm{Fc}$, or sialic acid-enriched $\mathrm{Fc}$ in coating buffer (0.05 M sodium carbonate buffer, $\mathrm{pH}$ 9). Binding efficiency to the plate was examined using anti-human IgG Fc-HRP as the probe. Wells were washed and incubated with human serum complement diluted in PBS containing 0.05\% Tween20 (PBS-T) and 1\% BSA for 2 hours at room temperature. After washing with PBS-T, wells were incubated for 1 hour with biotinylated anti-C1q, washed with PBS-T, and incubated for 1 hour with streptavidin-HRP. HRP activity was quantified using incubation TMB-ELISA substrate and colorimetric detection at $450 \mathrm{~nm}$.

Serum IgG quantification. Serum samples were taken before the initiation of the study (baseline) and 24 weeks (ICE trial) or $>7$ weeks (Marburg cohort) thereafter; samples were stored at $-80^{\circ} \mathrm{C}$. Total serum IgG levels were determined using a commercially available human IgG ELISA (Immundiagnostik).

Lectin immunoblotting and Fc-linked glycan profiling. Fc-linked glycans were analyzed by lectin immunoblotting using the biotinylated lectins SNA, ECL, LCA, and AAL (10, 11). Lectin binding was detected using HRP-labeled streptavidin and quantified by chemiluminescence using ImageJ. Signal intensities for each glycan (fucose, terminal galactose, and sialic acid) were normalized to the signal obtained for the core glycan (mannose).

SC5b-9 ELISA. Complement activation was determined in patients with CIDP from whom additional serum samples were available (Marburg cohort) using an ELISA detecting serum levels of the TCC (SC5b-9) according to the manufacturer's recommendation.

SPR. A Biacore T100 (GE Healthcare) was used for measuring kinetic data. Antibodies were immobilized on a CMD500L sensor chip surface via EDC/ NHS (0.4 M/ 0.1 M) surface activation in 10 $\mathrm{mM}$ acetate buffer pH 5.5 at $0.1 \mathrm{mg} / \mathrm{ml}(2,800 / 2,800 \mathrm{RU}$ for RTX/ tetra-Fc-sialylated RTX) as previously described (59). Dilution series of C1q ( $5 \mathrm{nM}-20 \mathrm{pM}$ ) were injected for 75 seconds at $30 \mu \mathrm{l} / \mathrm{min}$. Sensograms were analyzed using Biacore Evaluation Software applying a 1:1 kinetic model.

Statistics. All data represent the mean \pm SD. Statistical significance was defined at a $P$ value of 0.05 or less. All analyses were performed using Prism software, version 5 (GraphPad Software, Inc.). Specific statistical tests are listed in figure legends.

Study approval. The IRBs and ethics committees of the University of Marburg (file reference: 46/00) and all centers that participated in the ICE trial (32) approved the study. All patients provided written informed consent.

\section{Acknowledgments}

We thank our patients for their continuous cooperation. I. Quast was supported by a DOC scholarship provided by the Austrian Academy of Sciences (ÖAW). Christian W. Keller was supported by a postdoctoral fellowship provided by the German Research Foundation (DFG). We thank Patrick Weber (Institute of Experimental Immunology, Laboratory of Neuroinflammation, University of Zürich) for expert technical assistance and Jens Sobek (Functional Genomics Center, University of Zürich) for assistance with Biacore measurements. The work was supported in part by the German Science Foundation (DFG grant TRR130 to F. Nimmerjahn) and the NIH (NIH grant R01 GM096973 to L.X. Wang).

Address correspondence to: Jan D. Lünemann, Institute of Experimental Immunology, Laboratory of Neuroinflammation, University of Zürich, Winterthurerstrasse 190, CH-8057 Zürich, Switzerland. Phone: 41.44.635.3710; E-mail: jan.luenemann@uzh.ch. 
1. Schwab I, Nimmerjahn F. Intravenous immunoglobulin therapy: how does IgG modulate the immune system? Nat Rev Immunol. 2013;13(3):176-189.

2. Carroll MC. The role of complement and complement receptors in induction and regulation of immunity. Annu Rev Immunol. 1998;16:545-568.

3. Dalziel M, Crispin M, Scanlan CN, Zitzmann N, Dwek RA. Emerging principles for the therapeutic exploitation of glycosylation. Science. 2014;343(6166):1235681.

4. Barb AW, Prestegard JH. NMR analysis demonstrates immunoglobulin $\mathrm{G} \mathrm{N}$-glycans are accessible and dynamic. Nat Chem Biol. 2011;7(3):147-153.

5. Lux A, Nimmerjahn F. Impact of differential glycosylation on IgG activity. Adv Exp Med Biol. 2011;780:113-124.

6. Arnold JN, Wormald MR, Sim RB, Rudd PM, Dwek RA. The impact of glycosylation on the biological function and structure of human immunoglobulins. Annu Rev Immunol. 2007;25:21-50.

7. Parekh RB, et al. Association of rheumatoid arthritis and primary osteoarthritis with changes in the glycosylation pattern of total serum IgG. Nature. 1985;316(6027):452-457.

8. Parekh RB, Roitt IM, Isenberg DA, Dwek RA, Ansell BM, Rademacher TW. Galactosylation of IgG associated oligosaccharides: reduction in patients with adult and juvenile onset rheumatoid arthritis and relation to disease activity. Lancet. 1988;1(8592):966-969.

9. Fokkink WJ, et al. IgG Fc N-glycosylation in Guillain-Barre syndrome treated with immunoglobulins. J Proteome Res. 2014;13(3):1722-1730.

10. Kaneko Y, Nimmerjahn F, Ravetch JV. Antiinflammatory activity of immunoglobulin $\mathrm{G}$ resulting from $\mathrm{Fc}$ sialylation. Science. 2006;313(5787):670-673.

11. Anthony RM, Nimmerjahn F, Ashline DJ, Reinhold VN, Paulson JC, Ravetch JV. Recapitulation of IVIG anti-inflammatory activity with a recombinant IgG Fc. Science. 2008;320(5874):373-376.

12. Anthony RM, Wermeling F, Karlsson MC, Ravetch JV. Identification of a receptor required for the anti-inflammatory activity of IVIG. Proc Natl Acad Sci U S A. 2008;105(50):19571-19578.

13. Anthony RM, Kobayashi T, Wermeling F, Ravetch $\mathrm{JV}$. Intravenous gammaglobulin suppresses inflammation through a novel $\mathrm{T}(\mathrm{H}) 2$ pathway. Nature. 2011;475(7354):110-113.

14. Oefner CM, et al. Tolerance induction with $\mathrm{T}$ cell-dependent protein antigens induces regulatory sialylated IgGs. J Allergy Clin Immunol. 2012;129(6):1647-1655.

15. Hess $C$, et al. $T$ cell-independent $B$ cell activation induces immunosuppressive sialylated IgG antibodies. J Clin Invest. 2013;123(9):3788-3796.

16. Glennie MJ, French RR, Cragg MS, Taylor RP. Mechanisms of killing by anti-CD20 monoclonal antibodies. Mol Immunol. 2007;44(16):3823-3837.

17. Huang W, Giddens J, Fan SQ, Toonstra C, Wang LX. Chemoenzymatic glycoengineering of intact IgG antibodies for gain of functions. J Am Chem Soc. 2012;134(29):12308-12318.

18. Iida $S$, et al. Nonfucosylated therapeutic IgG1 antibody can evade the inhibitory effect of serum immunoglobulin $\mathrm{G}$ on antibodydependent cellular cytotoxicity through its high binding to FcgammaRIIIa. Clin Cancer Res. 2006;12(9):2879-2887.

19. Barb AW, Brady EK, Prestegard JH. Branchspecific sialylation of IgG Fc glycans by ST6Gal-I. Biochemistry. 2009;48(41):9705-9707.

20. Washburn N, et al. Controlled tetra-Fc sialylation of IVIg results in a drug candidate with consistent enhanced anti-inflammatory activity. Proc Natl Acad Sci U S A. 2015;112(11):E1297-E1306.

21. Kuenzle S, et al. Pathogen specificity and autoimmunity are distinct features of antigen-driven immune responses in neuroborreliosis. Infect Immun. 2007;75(8):3842-3847.

22. Dale RC, et al. Antibodies to MOG have a demyelination phenotype and affect oligodendrocyte cytoskeleton. Neurol Neuroimmunol Neuroinflamm. 2014;1(1):e12.

23. Holers VM. Complement and its receptors: new insights into human disease. Annu Rev Immunol. 2014;32:433-459.

24. Nimmerjahn F, Anthony RM, Ravetch JV. Agalactosylated IgG antibodies depend on cellular Fc receptors for in vivo activity. Proc Natl Acad Sci US A. 2007;104(20):8433-8437.

25. Koller H, Kieseier BC, Jander S, Hartung HP. Chronic inflammatory demyelinating polyneuropathy. N Engl J Med. 2005;352(13):1343-1356

26. Tackenberg B, et al. Classifications and treatment responses in chronic immune-mediated demyelinating polyneuropathy. Neurology. 2007;68(19):1622-1629.

27. Meyer zu Horste G, Hartung HP, Kieseier BC. From bench to bedside - experimental rationale for immune-specific therapies in the inflamed peripheral nerve. Nat Clin Pract Neurol. 2007;3(4):198-211.

28. Dalakas MC, Engel WK. Immunoglobulin and complement deposits in nerves of patients with chronic relapsing polyneuropathy. Arch Neurol. 1980;37(10):637-640.

29. Hays AP, Lee SS, Latov N. Immune reactive C3d on the surface of myelin sheaths in neuropathy. J Neuroimmunol. 1988;18(3):231-244.

30. Yan WX, Taylor J, Andrias-Kauba S, Pollard JD. Passive transfer of demyelination by serum or IgG from chronic inflammatory demyelinating polyneuropathy patients. Ann Neurol. 2000;47(6):765-775.

31. Dalakas MC. Mechanisms of action of IVIg and therapeutic considerations in the treatment of acute and chronic demyelinating neuropathies. Neurology. 2002;59(12 suppl 6):S13-S21.

32. Hughes RA, et al. Intravenous immune globulin ( $10 \%$ caprylate-chromatography purified) for the treatment of chronic inflammatory demyelinating polyradiculoneuropathy (ICE study): a randomised placebo-controlled trial. Lancet Neurol. 2008;7(2):136-144.

33. Mollnes TE, et al. Complement activation in patients with systemic lupus erythematosus without nephritis. Rheumatology (Oxford). 1999;38(10):933-940.

34. Dalakas MC, Medscape. Advances in the diagnosis, pathogenesis and treatment of CIDP. Nat Rev Neurol. 2011;7(9):507-517.

35. Baudino L, et al. Differential contribution of three activating IgG Fc receptors (Fc $\gamma \mathrm{RI}$, Fc $\gamma \mathrm{RIII}$, and Fc $\gamma$ RIV) to IgG2a- and IgG2b-induced auto- immune hemolytic anemia in mice. J Immunol. 2008;180(3):1948-1953.

36. Beers $\mathrm{SA}$, et al. Antigenic modulation limits the efficacy of anti-CD20 antibodies: implications for antibody selection. Blood.2010;115(25):5191-5201

37. Biburger $\mathrm{M}$, et al. Monocyte subsets responsible for immunoglobulin G-dependent effector functions in vivo. Immunity. 2011;35(6):932-944.

38. Clynes R, Ravetch JV. Cytotoxic antibodies trigger inflammation through $\mathrm{Fc}$ receptors. Immunity. 1995;3(1):21-26.

39. Fossati-Jimack L, et al. Markedly different pathogenicity of four immunoglobulin $\mathrm{G}$ isotypeswitch variants of an antierythrocyte autoantibody is based on their capacity to interact in vivo with the low-affinity Fcgamma receptor III. J Exp Med. 2000;191(8):1293-1302.

40. Hamaguchi Y, Xiu Y, Komura K, Nimmerjahn F, Tedder TF. Antibody isotype-specific engagement of $\mathrm{Fc} \gamma$ receptors regulates B lymphocyte depletion during CD20 immunotherapy. J Exp Med. 2006;203(3):743-753.

41. Hogarth PM. Fc receptors are major mediators of antibody based inflammation in autoimmunity. Curr Opin Immunol. 2002;14(6):798-802.

42. Kaneko Y, Nimmerjahn F, Madaio MP, Ravetch JV. Pathology and protection in nephrotoxic nephritis is determined by selective engagement of specific Fc receptors. JExp Med. 2006;203(3):789-797.

43. Nimmerjahn F, Bruhns P, Horiuchi K, Ravetch JV. FcgammaRIV: a novel FcR with distinct IgG subclass specificity. Immunity. 2005;23(1):41-51.

44. Nimmerjahn F, Ravetch JV. Divergent immunoglobulin g subclass activity through selective Fc receptor binding. Science. 2005;310(5753):1510-1512.

45. Ravetch JV, Clynes RA. Divergent roles for Fc receptors and complement in vivo. Annu Rev Immunol. 1998;16:421-432.

46. Sylvestre D, Clynes R, Ma M, Warren H, Carroll MC, Ravetch JV. Immunoglobulin G-mediated inflammatory responses develop normally in complement-deficient mice. J Exp Med. 1996;184(6):2385-2392.

47. Sylvestre DL, Ravetch JV. Fc receptors initiate the Arthus reaction: redefining the inflammatory cascade. Science. 1994;265(5175):1095-1098.

48. Takai T, Li M, Sylvestre D, Clynes R, Ravetch JV. FcR gamma chain deletion results in pleiotrophic effector cell defects. Cell. 1994;76(3):519-529.

49. Uchida J, et al. The innate mononuclear phagocyte network depletes B lymphocytes through Fc receptor-dependent mechanisms during anti-CD20 antibody immunotherapy. J Exp Med 2004;199(12):1659-1669.

50. Duncan AR, Winter G. The binding site for C1q on IgG. Nature. 1988;332(6166):738-740.

51. Idusogie EE, et al. Mapping of the C1q binding site on rituxan, a chimeric antibody with a human IgG1 Fc. JImmunol. 2000;164(8):4178-4184.

52. Ward ES, Ghetie V. The effector functions of immunoglobulins: implications for therapy. Ther Immunol. 1995;2(2):77-94.

53. Sondermann P, Pincetic A, Maamary J, Lammens K, Ravetch JV. General mechanism for modulating immunoglobulin effector function. Proc Natl Acad Sci U S A. 2013;110(24):9868-9872.

54. Ahmed AA, et al. Structural characterization of 


\section{RESEARCH ARTICLE}

anti-inflammatory immunoglobulin G Fc proteins. J Mol Biol. 2014;426(18):3166-3179.

55. Tackenberg B, et al. Impaired inhibitory Fc $\gamma$ receptor IIB expression on B cells in chronic inflammatory demyelinating polyneuropathy. Proc Natl Acad SciUS A. 2009;106(12):4788-4792.

56. Harre U, et al. Glycosylation of immunoglobulin
$G$ determines osteoclast differentiation and bone loss. Nat Commun. 2015;6:6651.

57. Lee-MacAry AE, et al. Development of a novel flow cytometric cell-mediated cytotoxicity assay using the fluorophores PKH-26 and TO-PRO-3 iodide. J Immunol Methods. 2001;252(1-2):83-92.

58. Lux A, Yu X, Scanlan CN, Nimmerjahn F. Impact
The Journal of Clinical Investigation

of immune complex size and glycosylation on IgG binding to human Fc $\gamma$ Rs. J Immunol. 2013;190(8):4315-4323.

59. Dall'Acqua WF, Cook KE, Damschroder MM, Woods RM, Wu H. Modulation of the effector functions of a human IgG1 through engineering of its hinge region. JImmunol. 2006;177(2):1129-1138. 